\title{
Segmental dynamics and the correlation length in nanoconfined PMMA
}

\author{
R. Casalini ${ }^{\mathrm{a}}$, L. Zhu ${ }^{\mathrm{b}}$, E. Baer ${ }^{\mathrm{b}}$, C.M. Roland ${ }^{\mathrm{a}}$ \\ ${ }^{a}$ Naval Research Laboratory, Chemistry Division, Code 6120, Washington DC 20375-5342 \\ ${ }^{b}$ Department of Macromolecular Science and Engineering, Case Western Reserve University, Cleveland, \\ OH 44106-7202
}

(February 3 , 2016)

\begin{abstract}
We studied the segmental dynamics of poly(methyl methacrylate) (PMMA) in multilayered films with polycarbonate (PC) having layer thicknesses as small as $4 \mathrm{~nm}$. Such a design provides macroscopic dimensions enabling macroscopic measurements, while maintaining nmscale confinement. Of particular significance, we were able to determine correlation length scales for the polymer under nanoconfinement. Increases of the local segmental relaxation time, $\tau_{\alpha}$, and glass transition temperature, $T_{g}$, were observed with decreasing layer thickness. However, neither the fragility ( $T_{g}$-normalized temperature dependence of $\left.\tau_{\alpha}\right)$ nor the breadth of the relaxation dispersion were affected by the geometric confinement. More significantly, the dynamic correlation volume, a measure of the degree of cooperativity of the dynamics, was also unaffected; that is, values of the correlation volume for confined PMMA were equal to those of the bulk polymer when compared at the same $\tau_{\alpha}$. This absence of an effect of geometric confinement on dynamic correlation, even when the confinement length scale approaches the correlation length scale, suggests a nonspherical correlation volume. The slowing of the segmental dynamics of PMMA confined to thin layers is due to admixing of the high $T_{g}$ polycarbonate. A negligible mixing enthalpy gives rise to an extended interfacial region, which comprises a significant fraction of each layer.
\end{abstract}




\section{Introduction}

The dynamics of polymers approaching their glass transition is characterized by an increase of the segmental relaxation time, $\tau_{\alpha}$, by several orders of magnitude over a small temperature range. This dramatic slowing down arises from the cooperative nature of the local segmental motions. Increasingly upon cooling, the segments cannot reorient or diffuse independently, so that motion of each segment becomes more coupled to that of a number of neighboring segments. The number of correlated units, $N_{\mathrm{c}}$, grows in concert with the slowing of the dynamics reflected in the increase in $\tau_{\alpha}$. Thus, quantifying the extent of these dynamic correlations is essential to characterizing motions in dense, complex systems. This cooperativity can be associated with a correlation length, $\xi$, that has a lower bound obtained from the assumption of spherical cooperative regions:

$$
\xi=\left(v_{m} N_{c}\right)^{\frac{1}{3}}
$$

where $v_{\mathrm{m}}$ is the volume of a chain segment. On approaching the glass transition, $N_{\mathrm{c}}$ for polymers attains values on the order of hundreds [1], so from eq.(1) $\xi$ can be several nanometers. This suggests that when the dimensions of a polymer film are of this order, the geometrical constraint may affect the dynamics and related properties, such as the glass transition temperature, ionic conductivity, dielectric loss, etc.

Since the pioneering experiments of Jackson and McKenna [2] and Keddie et al. [3], a variety of experimental techniques have revealed a change in the glass transition temperature, $T_{g}$, for confined liquids and polymers. However, due to the non-negligible effect of the interfaces (free or interacting), the available results do not always agree concerning even the qualitative nature of this confinement effect. [4,5,6,7] The apparent discrepancies usually are ascribed to the effect of free surfaces on mobility, which complicates interpretation of true confinement effects. And while dynamic correlation is generally accepted as the cause, or at least being strongly related to the slowed dynamics that lead to glass formation [8], there has been no experimental determination of the effect, if any, of nanoconfinement on $N_{c}$. This is an important question, given the correspondence between the changes in $\tau_{\alpha}$ and $N_{\mathrm{c}}$ predicted by the Adam-Gibbs model [9] and the more recent random first order transition theory [10], as discussed also in [1]. Experiments have confirmed the close connection between $N_{c}$ and $\tau_{\alpha}[11,12]$. 


\section{Materials and methods}

In this work we used dielectric spectroscopy to probe the dynamics for poly(methyl methacrylate) (PMMA) nanometer layers confined between similarly sized layers of poly(bisphenol A carbonate) (PC). Our measurements were carried out between the glass transition temperatures of the bulk polymers, $T_{g}=388 \mathrm{~K}$ for PMMA and $420 \mathrm{~K}$ for PC. The samples were composed of 1024 alternating layers of PMMA and PC. The total film thickness was $4.0,8.2$ or $25.6 \mu \mathrm{m}$, with all layers in a given sample having the same thickness, 4,8 or 25 $\mathrm{nm}$ respectively. For dielectric measurements aluminum electrodes (diameter 12.8mm) were deposited under vacuum on both surfaces of the films (the thickness variation over a sample was $0.2 \mu \mathrm{m})$. These multilayer films were prepared by melt co-extrusion, using a forced assembly technique developed by Baer and coworkers [13,14,15]. Unlike conventional thin films [16, 17], the macroscopic size of these multilayer films makes possible modulated scanning calorimetry (MDSC) to determine the complex heat capacity, supplementing the dielectric measurements. The ability to measure both dynamic and thermodynamic properties allows two independent determinations of the dynamic correlation length. In a related approach, Hayashi and Fukao [18] stacked multiple $(\leq 300)$ thin films of glassy PMMA. This yields samples of sufficient bulk for calorimetry, while still exhibiting thin film dynamics. However, such interfaces are different from herein, since our layering is done with the components in the melt state, yielding near equilibrium morphologies.

To obtain $N_{c}(T)$ we applied the method of Berthier et al. [19]

$$
N_{c}(T)=\max \left\{\chi_{4}(t)\right\} \cong \frac{k T^{2}}{c_{p}}\{\max \partial C(t) / \partial t\}^{2}
$$

in which $\chi_{4}$ is a four point correlation function, $c_{p}$ is the configurational heat capacity, $k$ the Boltzmann constant, and $C(t)$ a linear correlation function, measured herein by dielectric spectroscopy. When $C(t)$ has the stretched exponential form,

$$
C(t)=\exp \left\{-\left[t / \tau_{\alpha}(T)\right]^{\beta_{K W W}(T)}\right\}
$$

with a $\beta_{K W W}$ a $T$-dependent constant, eq.(2) reduces to [1] 


$$
N_{c}(T) \cong \frac{k T^{2}}{m_{0} \Delta C_{p}} \frac{\beta_{K W W}(T)^{2}}{e^{2}}\left(\frac{d \ln \left(\tau_{\alpha}\right)}{d \ln (T)}\right)^{2}
$$

where $e$ is Euler's number, $m_{0}$ the repeat unit molecular weight ( $=110.12 \mathrm{~g} / \mathrm{mol}$ for PMMA), and the configurational heat capacity is estimated as $\Delta C_{p}$, the difference between $\mathrm{C}_{p}$ of the melt and the glass. Potential pitfalls in the application of this method, widely applied for the quantification of dynamic correlation [1,4], have been discussed [12].

\section{Results and discussion}

The dielectric loss spectrum of PMMA, $\varepsilon_{P M M A}^{\prime \prime}$, is characterized by two dispersions, the segmental relaxation $(\alpha)$ and a prominent secondary $(\beta)$ process. To compare the spectra of the multilayered sample with those of the bulk PMMA, the dielectric response of the laminate has to be corrected for the contribution of the PC. Since the layers are in series, the complex permittivity is given by $\varepsilon_{P M M A}^{*}=\phi\left[1 / \varepsilon_{\text {Total }}^{*}-(1-\phi) / \varepsilon_{P C}^{*}\right]^{-1}$, where the volume fraction $\phi=0.5$. Absent this correction the loss peak artificially shifts to higher frequency. The corrected spectrum at $T=406.8 \mathrm{~K}$ is shown in Figure 1, where it can be seen that for $8 \mathrm{~nm}$ layers, the $\alpha$-relaxation is at a lower frequency than for bulk PMMA (measured at $0.2 \mathrm{~K}$ lower temperature, 406.6 K); on the other hand, the $\beta$ relaxation dynamics are unaffected by the confinement. A systematic increase of the dielectric strength of both processes with decreasing layer thickness is also evident in the spectra; the effect is larger for the $\alpha$ than for the $\beta$ relaxation. This indicates more orientation of the polar segments by the field, perhaps due to suppression of dipole fluctuations from the confinement or possibly a different chain orientation than for the bulk.

The relaxation times were extracted by fitting a linear superposition of relaxation functions to the experimental loss spectrum, with the Laplace transform of eq.(3) used for the $\alpha$-peak and the Havriliak-Negami equation [20] for the $\beta$ relaxation. Previously we showed that the method used to deconvolute the two relaxations in PMMA does not influence the values obtained for $\tau_{\alpha}$ and $\tau_{\beta}[21]$ 


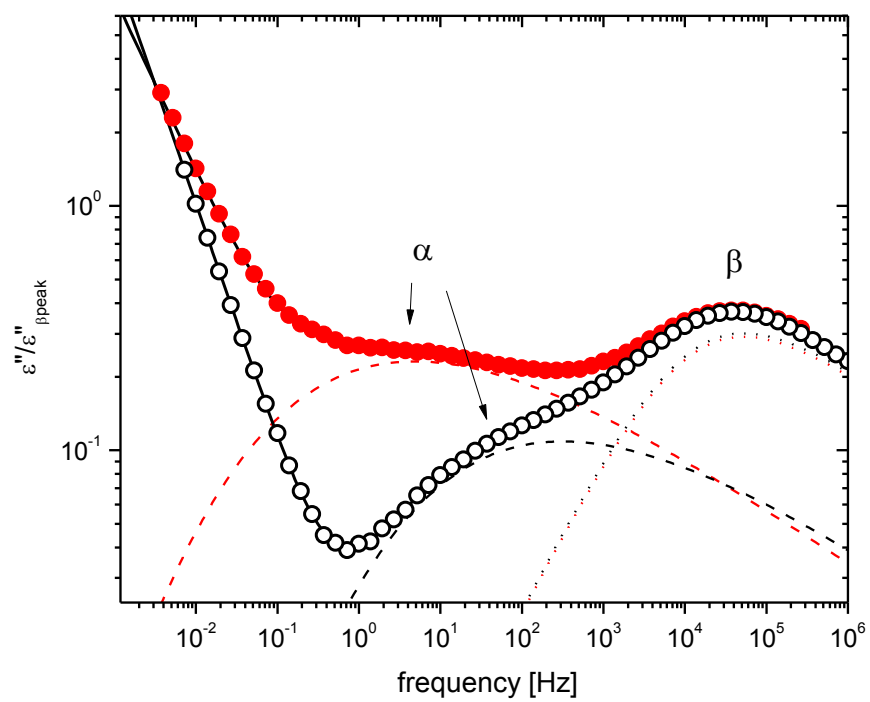

Figure 1. Dielectric loss spectra for bulk PMMA (solid circles; $\mathrm{T}=406.6 \mathrm{~K}$ ) and multilayered PMMA with $8 \mathrm{~nm}$ layer thickness (open circles; $\mathrm{T}=406.8 \mathrm{~K}$ ). For the multilayer sample, the permittivity of the PMMA was extracted from the measured permittivity by correcting for the PC contribution as discussed in the text. There is a significant slowing down of the $\alpha$ process under confinement, whereas the $\beta$ peak frequency is unaffected. The ordinate values were normalized by the maximum of the $\beta$ peak. The rise of the dielectric loss at low frequency reflects conduction loss from ionic impurities. The dashed and dotted lines are the respective contributions of the $\alpha$ and $\beta$ process as obtained from the fit.

Table 1. Relaxation parameters for PMMA in bulk and under nanoconfinement.

\begin{tabular}{|l|c|c|c|c|}
\hline & bulk & $25 \mathrm{~nm}$ & $8 \mathrm{~nm}$ & $4 \mathrm{~nm}$ \\
\hline $\mathrm{T}\left(\tau_{\alpha}=10 \mathrm{~s}\right)[\mathrm{K}]$ & $390.3 \pm 0.5$ & $395.8 \pm 0.5$ & $398.5 \pm 0.5$ & $398.4 \pm 0.5$ \\
\hline $\mathrm{MDSC}_{\mathrm{g}}[\mathrm{K}]$ & $387 \pm 1$ & $393 \pm 1$ & $394 \pm 1$ & $395 \pm 1$ \\
\hline $\mathrm{N}_{\mathrm{c}}\left(\tau_{\alpha}=10 \mathrm{~s}\right)$ eq. $(4)$ & $378 \pm 13$ & $364 \pm 15$ & $377 \pm 16$ & $395 \pm 20$ \\
\hline$\xi=\left(v_{\mathrm{m}} \mathrm{N}_{\mathrm{c}}\right)^{1 / 3}\left(\tau_{\alpha}=10 \mathrm{~s}\right)$ & $3.8 \pm 0.2$ & $3.7 \pm 0.2$ & $3.8 \pm 0.2$ & $3.8 \pm 0.2$ \\
\hline $\mathrm{N}_{\mathrm{c}}\left(\tau_{\alpha}=250 \mathrm{~s}\right)$ eq. $(6)$ & $50 \pm 7$ & $40 \pm 6$ & $42 \pm 6$ & $38 \pm 9$ \\
\hline$\xi=\left(v_{\mathrm{m}} \mathrm{N}_{\mathrm{c}}\right)^{1 / 3}\left(\tau_{\alpha}=250 \mathrm{~s}\right)$ & $1.9 \pm 0.1$ & $1.8 \pm 0.1$ & $1.8 \pm 0.1$ & $1.7 \pm 0.1$ \\
\hline
\end{tabular}

$v_{\mathrm{m}}=86 \mathrm{~mL} / \mathrm{mol}\left(0.14 \mathrm{~nm}^{3}\right.$ per repeat unit $)$ 


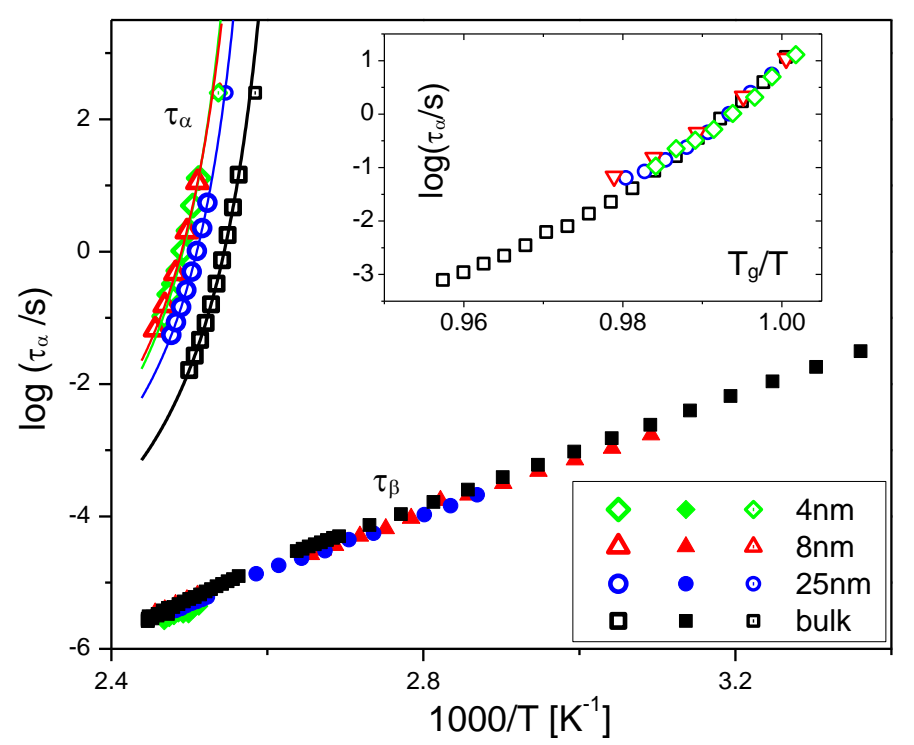

Figure 2. Arrhenius plot of the dielectric relaxation times for bulk PMMA (squares) and three PMMA multilayers (diamonds $-4 \mathrm{~nm}$ layer thickness; triangles $-8 \mathrm{~nm}$; circles $-25 \mathrm{~nm}$ ). Open symbols are for the $\alpha$ relaxation and filled symbols for the $\beta$ relaxation. The $\alpha$ relaxation time from MDSC for each multilayer sample is also shown (dotted symbols at $\log \tau_{\alpha}=2.40$ ). In the inset are $\tau_{\alpha}$ versus inverse $T_{g^{-}}$ normalized temperature, using $\tau_{\alpha}\left(T_{g}\right)=10 \mathrm{~s}$, showing that the fragilities of the four samples are essentially the same.

The respective $\tau_{\alpha}$ and $\tau_{\beta}$ for bulk PMMA and three multilayers $(4,8$ and $25 \mathrm{~nm}$ layer thickness) are displayed in Figure 2. The secondary relaxation times are invariant to confinement, which is unsurprising given the local nature of the $\beta$-process. However, we observe an increase of $\tau_{\alpha}$ for confined PMMA; for example, at $8 \mathrm{~nm}$ thickness, the $\alpha$-relaxation time is about three orders of magnitude larger than for the bulk polymer. The Vogel-Fulcher equation

$$
\tau_{\alpha}=\tau_{0} \exp \left[\frac{B}{T-T_{0}}\right]
$$

with $\tau_{0}, B$, and $T_{0}$ constants, was fit to the $\tau_{\alpha}$, recognizing that the limited span of the data makes these fits somewhat imprecise. The $T_{g}$-normalized temperature dependence ("fragility"), $\left.\frac{d \log \left(\tau_{\alpha}\right)}{d\left(T_{g} / T\right)}\right|_{T=T_{g}}$, is the same for all samples within the (large) scatter; using $T\left(\tau_{\alpha}=10 \mathrm{~s}\right)$ to define $T_{g}$, we obtain $139 \pm 8$. This differs from thin polymer films formed by layering PMMA in the glassy state; such multilayers exhibit changes of the fragility from the bulk [18]. There is no 
apparent change in the $\alpha$ peak shape due to confinement, $\beta_{K W W}=0.24 \pm 0.02$ at $T_{g}$, although the overlap with the high-frequency $\beta$ peak (Fig. 1) introduces some uncertainty into the fits to the spectra. An invariant $\beta_{K W W}$ is consistent with the observed constant fragility.

MDSC measurements showed similar changes in the glass transition of the confined PMMA, increases of 5.7 and $6.9 \mathrm{~K}$ for 25 and $8 \mathrm{~nm}$ confinement, respectively (Table 1). The modulation frequency was $0.025 \mathrm{~Hz}$, so that the MDSC $T_{g}$ corresponds to an $\alpha$ relaxation time of 250 s. Extrapolations of the dielectric $\tau_{\alpha}$ are consistent with these MDSC results (Fig. 2).

From eq.(4) we calculate the number of correlating repeat units, using $\Delta C_{p}$ from MDSC, along with the dielectric relaxation results. Confinement strongly increases $N_{\mathrm{c}}$ at each temperature (see Figure 3 inset), by almost a factor of four going from bulk to $8 \mathrm{~nm}$ PMMA layers. Interestingly, the confinement effect seems to saturate at $8 \mathrm{~nm}$, since for thinner layers (4 $\mathrm{nm}$ ) the dielectric relaxation times are the same as for $8 \mathrm{~nm}$. However, when plotted as a function of $\tau_{\alpha}$ (Fig. 3), there is no significant change in $N_{\mathrm{c}}\left(\tau_{\alpha}\right)$ among the four samples. This correspondence supports the idea that dynamic correlation governs the $\alpha$-relaxation dynamics $[11,12,9,10,22]$. A lower bound for the correlation length can be obtained from the data in Fig. 3 using eq.(1) and the segmental volume at $T_{g}$. (This is an approximation because the equation of state for confined PMMA is lacking; however, the error is only a few percent and thus inconsequential for our purposes.) We obtain $\xi \geq 4 \mathrm{~nm}$ at $T_{g}$, and $\xi \geq 1 \mathrm{~nm}$ at the highest measurement temperature (for which $\tau_{\alpha} \sim 50 \mathrm{~ms}$ ). These values are quite small: the lower bound on the correlation length is comparable with the confined layer thickness for the thinner layers. The implication is that geometrical encroachment on the correlation volume does not mitigate the dynamic cooperativity; consequently, slowing of the dynamics can occur (due to some other mechanism).

We note that the application of eq.(4) has been found to yield disparate results when applied to high pressure measurements on different materials by different groups [12]; however, the trends obtained herein, based on a consistent treatment of data for the same polymer at ambient pressure, should be reliable. Nevertheless, it is useful to verify the $N_{c}$ using another method. Donth [23] proposed extracting the number of correlating units directly from the breadth of the glass transition, $\delta T$, in calorimetry measurements 


$$
N_{c}(T)=\frac{R T^{2} \Delta c_{p}^{-1}}{m_{0}\left(\delta T^{2}\right)}
$$

For the $8 \mathrm{~nm}$ layers, $\delta T=6.6 \pm 0.71 \mathrm{~K}$ and $\Delta C_{p}^{-1}=0.107 \pm 0.005 \mathrm{Jg}^{-1} \mathrm{~K}^{-1}$ at the calorimetric $T_{\mathrm{g}}$, for which the dielectric $\tau_{\alpha} \sim 250 \mathrm{~s}$. This yields $N_{c}=42 \pm 6$, and a lower bound for $\xi$ equal to $1.9 \pm 0.3$ $\mathrm{nm}$. This is smaller than the value estimated from the dielectric measurements (eq. (4)), very similar to the differences between the two methods when applied to bulk PMMA [1,24]. DalleFerrier et al. [25] have pointed out that the precision in the measurement of correlation volumes is highest for dielectric spectroscopy; thus, the larger $N_{\mathrm{c}}$ herein are more reliable. Both determinations of $\xi$, of course, rely on assumptions, and lacking any direct experimental means to determine $\xi$, we cannot choose between eqs. (4) and (6). Most relevant, however, is that both experiments yield $\xi$ that are on the order of the layer size for the thinner samples, affirming that in these experiments geometric confinement does not affect the dynamics even when the confinement is commensurate with the cooperative length scale.

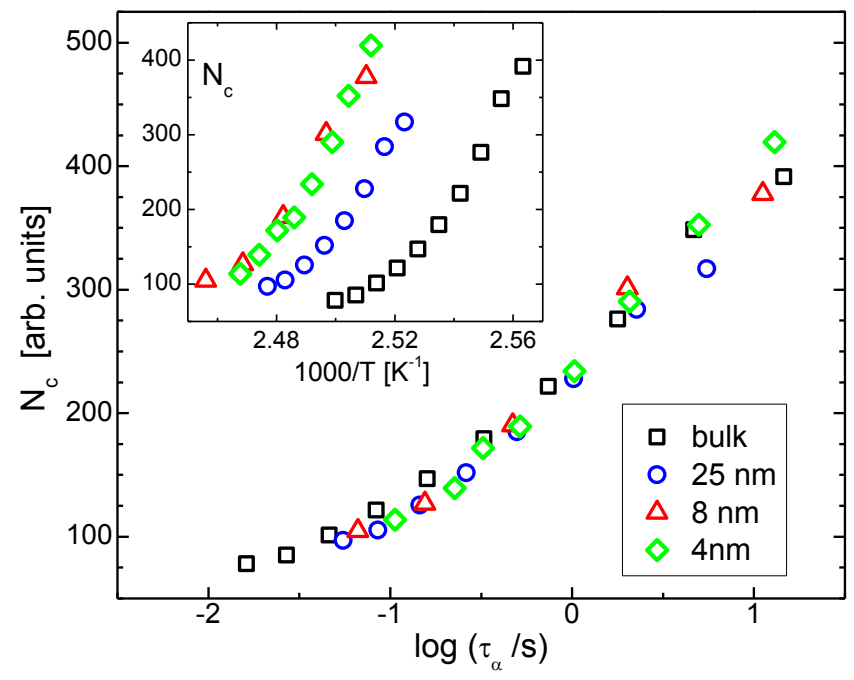

Figure 3. Number of dynamically correlated units versus the $\alpha$ relaxation time for bulk and nanoconfined PMMA samples. Notwithstanding the large changes of $N_{\mathrm{c}}$ due to confinement (inset), the dependence of $N_{\mathrm{c}}$ on $\tau_{\alpha}$ is the same as for the bulk PMMA. Error bars are smaller than the symbol size. 


\section{Conclusions}

Our main result is the finding that the relationship between $\tau_{\alpha}$ and $N_{c}$ is unaltered, even for layer thicknesses of the PMMA comparable to $\xi$. Although segmental relaxation in $4 \mathrm{~nm}$ multilayers is three orders of magnitude slower than for bulk PMMA, $\xi$ (and $N_{\mathrm{c}}$ ) is the same as in the bulk polymer when compared at equal $\tau_{\alpha}$. This absence of a confinement effect on the cooperativity is surprising since the values of $\xi$ (Table 1) are equal to or smaller than the layer thickness. Thus, the dynamic correlation length does not appear to be limited by the geometric confinement, a result consistent with nonspherical cooperative regions. Such asymmetry calls to mind the string-like shape seen in molecular dynamics simulations [26,27], although the latter are for higher frequencies than the measurements herein. A non-spherical cooperative region seems to be at odds with the compact shape envisioned in the RFOT model [28]. Note that is has been shown recently by MD of polymer thin films [27] that independently of the interactions at the interfaces, the correlation between the polymer dynamics and $\xi$ remains unchanged, consistent with our results (Figure 3).

While geometrical encroachment on the correlation volume does not countervail the slowing down of the dynamics and consequent increase of $T_{g}$, the issue remains concerning the mechanism for the larger $\tau_{\alpha}$. One possibility is densification in the multilayers relative to the bulk. However, a previous study of very similar PC/PMMA multilayers [R.Y. F. Liu, Y. Jin, A.Hiltner, E. Baer, Macromol. Rapid Commun. 2003, 24, 943-948] found only modest increases in density $(<0.2 \%)$. From the known dependence of $T_{g}$ of PMMA on density [R. Casalini; C.M. Roland; S. Capaccioli, J. Chem. Phys. 126, 184903 (2007)], the effect on the glass transition temperature would be quite small $(\sim 0.3 \mathrm{~K})$.

Thus, we ascribe the increase in $T_{g}$ and $\tau_{\alpha}$ to interfacial interactions involving the glassy polycarbonate. Such an effect has been described previously [29;30]. The dispersive interfacial energy for the PMMA and PC can be estimated from the solubility parameters

$$
E=v_{m}\left(\delta_{P M M A}-\delta_{P C}\right)^{2}
$$

. The interaction parameters for the two polymers are nearly equal, $\delta_{P M M A}=20.48 \pm 2.2 \mathrm{MPa}^{1 / 2}$ [31] and $\delta_{P C}=20.56 \mathrm{MPa}^{1 / 2}$ [32]. Thus, from eq. (7) the interfacial energy is vanishingly small, $E \sim 10^{-4} R T$. This means the enthalpic barrier to mixing is negligible, and since the interfacial 
thickness varies inversely with interfacial energy $[33,34]$, the interphase in these samples is quite extended (as much as a few nm [35,36,37]). Robertson et al. [38] found that in polystyrene block copolymers, a small solubility parameter difference caused very large changes in $T_{g}$, several tens of K, while a large solubility parameter difference has been found to result in negligible changes in $T_{g}$ [39]. Herein, the presence of the PC within the interfacial region slows down the segmental dynamics of PMMA, significantly increasing its $T_{g}$. (Note that the polymers are not actually miscible, as seen from the observation of distinct glass transitions for both.) Recent simulations suggests that interfacial mixing is not a necessity for large changes of $T_{\mathrm{g}}$ under nanoconfinement. Such changes could be a consequence of interfacial interaction alone, depending on the rigidity of the confinement [40]. If the multilayer film were constructed by laminating glassy polymers, the interface would be sharp, and the behavior likely quite different [18].

\section{Acknowledgement}

The work at NRL was supported by the Office of Naval Research, in part by code 332 .

\section{References}

[1] S. Capaccioli, G.Ruocco, F. Zamponi, J. Phys. Chem. B, 112 (2008) 10652-10658.

[2] C.L. Jackson, G.B. McKenna, J. Non-Cryst. Sol. 131 (1991) 221-224; ibid, Chem. Mater. 8 (1996) 2128- 2137.

[3] J.L. Keddie, R.A.L. Jones, R.A. Cory, Europhys. Lett. 27 (1994) 59-64; J.L. Keddie, R.A.L. Jones, R.A. Cory, Faraday Discussions 98 (1994) 219-230.

[4] C.M. Roland, Macromolecules 43 (2010) 7875-7890.

[5] F. Kremer, et al. Eds. Broadband Dielectric Spectroscopy, Springer-Verlag, Berlin, Heidelberg, 2003.

[6] C. Schick and E. Donth, Physica Scripta 43 (1991) 423.

[7] M.Beiner and H.Huth, Nature Materials 2 (2003) 595.

[8] L. Berthier, G. Biroli, J.-P. Bouchaud, L. Cipelletti and W. van Saarloos, Dynamical Heterogeneities in Glasses, Colloids and Granular Materials, Oxford University Press, 2011.

[9] G. Adam, J.H. Gibbs, J. Chem. Phys. 43 (1965) 139-146.

[10] G. Biroli, J.-P. Bouchaud, in "Structural Glasses and Supercooled Liquids", Ed. P. G. Wolynes \& V. Lubchenko, John Wiley \& Sons, Inc., 2012, pp 31-113.

[11] D. Fragiadakis, R. Casalini, C.M. Roland, J. Phys. Chem. B 113 (2009) 13134-13137. 
[12] R. Casalini, D. Fragiadakis, C.M. Roland, J. Chem. Phys. 142 (2015) 064504.

[13] A. Flores, C. Arribas, F. Fauth, D. Khariwala, A. Hiltner, E. Baer, Polymer 51 (2010) 4530-4539.

[14] I.P. Orench, N. Stribeck, F. Ania, E. Baer, A. Hiltner, F.J. Balta-Calleja, Polymer 50(2009) 26802687.

[15] H.P. Wang, J.K. Keum, A. Hiltner, E. Baer, B. Freeman, A. Rozanski, Science 323 (2009) 757-760.

[16] R.D. Priestley, L.J. Broadbelt, J.M. Torkelson, K. Fukao, Phys. Rev. E 75 (2007) 061806.

[17] C.M. Evans, H. Deng, W.F. Jager, J.M. Torkelson Macromolecules 46(2013) 6091-6103.

[18] T. Hayashi, K. Fukao, Phys. Rev. E 89 (2014) 022602.

[19] L. Berthier, G. Biroli, J.P. Bouchaud, L. Cipelletti, D. El Masri, D. L’Hote, F. Ladieu, M. Pierno, Science 310 (2005) 1797-1800.

[20] C.M. Roland, Viscoelastic Behavior of Rubbery Materials, Oxford Univ. Press: Oxford UK, 2011.

[21] R. Casalini, A. Snow, C.M. Roland, Macromolecules 46 (2013) 330-334.

[22] H. Huth, M. Beiner, E. Donth, Phys Rev B 61 (2000) 15092

[23] E. Donth, J. Non-Cryst. Solids 53 (1982) 325-330.

[24] E. Donth, M. Beiner, S. Reissig, J. Korus, F. Garwe, S. Vieweg, S. Kahle, E. Hempel, K. Schroter, Macromolecules 29 (1996) 6589-6600.

[25] C. Dalle-Ferrier, C. Thibierge, C. Alba-Simionesco, L. Berthier, G. Biroli, J.-P. Bouchaud, F.

Ladieu, Phys. Rev. E 76 (2007) 041510.

[26] N. Lacevic, F.W. Starr, T.B. Schrøder, S.C. Glotzer, J. Chem. Phys. 199 (2003) 7372-7387 .

[27] P.Z. Hanakata, J.F. Douglas, F.W. Starr, Nat. Commun. 5 (2014) 4163.

[28] Stevenson, J.D.; Schmalian, J. and Wolynes, P.G. Nature Physics 2 (2006) 268-274.

[29] T. Babur, J. Balko, H. Budde, M. Beiner, Polymer 55 (2014) 6844.

[30] S. Pankaj and M. Beiner, J. Phys. Chem. B 114 (2010) 15459.

[31] Average of two values reported by E.A. Grulke in Polymer Handbook, $4^{\text {th }}$ Edition, Ed. J. Brandrup, E.H. Immergut, and E.A. Grulke, Wiley (1999).

[32] N. Muruganandam, W.J. Koros, D.R. Paul, J. Polym. Sci. Polym. Phys. Ed. 25 (1987) 1999-2026.

[33] E. Helfand, Y. Tagami, J. Chem. Phys. 56 (1972) 3592-3601.

[34] E. Helfand, Z.R. Wasserman, Macromolecules 9 (1976) 879-888. 
[35] R.Y.F. Liu, T. E. Bernal-Lara, A. Hiltner, and E. Baer, Macromolecules 37 (2004) 6972-6979.

[36] R.Y.F. Liu, A.P. Ranade, H.P.Wang, T.E. Bernal-Lara, A. Hiltner, E. Baer, Macromolecules 38 (2005) 10721-10727 .

[37] K. Arabeche, L. Delbreilh, R. Adhikari, G.H. Michler, A. Hiltner, E. Baer, J.-M. Saiter, Polymer 53 (2012) 1355-1361.

[38] C.G. Robertson, T.E. Hogan, M. Rackaitis, J.E. Puskas, X. Wang, J. Chem. Phys. 132 (2010) 104904.

[39] R. Casalini, D. Prevosto, M. Labardi, C.M. Roland ACS MacroLetters 4 (2015) 1022.

[40] R.J. Lang, W.L. Merling, D.S. Simmons, ACS Macro Lett. 3 (2014) 758-762. 
Graphical abstract: "Segmental dynamics and the correlation length in nanoconfined PMMA” R. Casalini, L. Zhu, E. Baer, C.M. Roland.

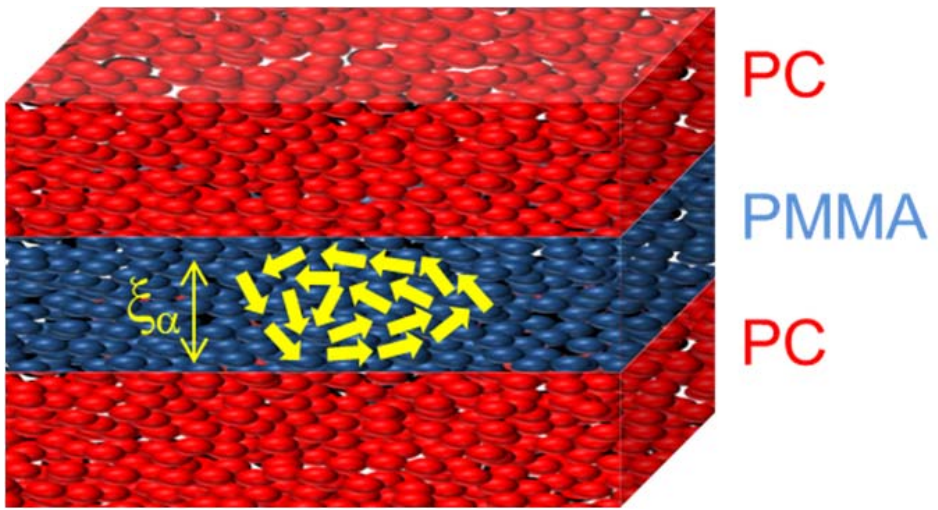

